509. K. A uwers und Th. Maas: Ueber das Dibrom-m-oxypseudocumylbromid.

(Eingegangen am 28. November.)

Ueber das isomere Pseudocumenoltribromid vom Schmp. $128^{\circ}$ und einige seiner Umwandlangsproducte ist bereits kurz berichtet worden 1). Die damaligen Angaben werden durch die nachsteheude Mittheilung ergänzt; ausserdem werden in ibr eine Anzahl weiterer Versuche beschrieben, die zur Ermittelung der Constitution des neuen Tribromids angestellt wurden. Wie bereits in der vorauggehenden theoretischen Abhandlung auseinandergesetzt worden ist, geht aus ihnen mit Sicherheit hervor, dass dem isomeren Tribromid die Formel

$$
{ }_{\mathrm{OH}}^{\mathrm{Br}} \overbrace{\mathrm{CH}}^{\mathrm{CH}_{3} \mathrm{Br}}
$$

zukommt, in ihm also das Dibrom-m-oxypseudocumylbromid vorliegt.

Der Mittheilung der betreffenden Experimente seien noch ein paar Worte über das Ergebniss einiger Reductionsversuche vorausgeschickt, das von allgemeinerem Interesse ist.

Es wurde beabsichtigt, verschiedene Umwandlungsproducte des Tribromids durch energische Reduction in bromfreie Verbindungen überzuführen, da deren Constitution voraussichtlich leichter festzustellen war, als die der bromhaltigen Producte. In erster Linie stellte man derartige Versuche mit der bei $103-104^{\circ}$ schmelzenden Diacetylverbindung,

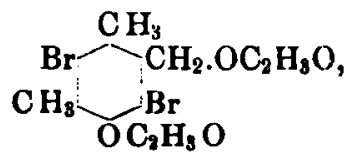

an, die das Ausgangamaterial für das Tribromid und alle weite. ren Derivate bildete. Als Reductionsmittel wurde erst Natriumamalgam, spăter Zinkstaub benutzt; als Lōsungsmittel 75 - procentige Essigsãure. Im ersten Falle wurde in der Kălte, im zweiten in der Siedehitze gearbeitet.

Es stellte sich nun heraus, dass unter diesen verschiedenen Bedingungen regelmässig nur das eine der beiden Bromatome herausgenommen wurde, obwohl die Versuche mehrfach wiederholt und verschieden lange im Gang erhalten wurden. Der einzige Untersehied bestand darin, dass bei den Reductionsversuchen in der Kalte eine einfach bromirte $\mathrm{Diacetyl}$ erbindung erbalten wurde, wäbrend bei

j) Diese Berichte 32, 17. 
den Versuchen in der Hitze ein einfach gebromtes, alkalilösliches Mono acetat entstand, die am Phenolsauerstoff haftende Acetylgruppe also durch die kochende wasserhaltige Essigsäure abgespalten worden war. Beide Substanzen liessen sich durch Verseifung in denselben Monobromphenolalkohol überführen; anch konnte das Monoacetat durch Kochen mit Essigsanreanhydrid in das Diacetat umgewandelt werden.

Die beiden Bromatome der ursprünglichen Diacetylverbindung haften also ungleich fest am Benzulkern. Bekanntlich werden Phenole sehr leicht in 0 - und $p$-Bromderivate übergeführt, während sich ihre $m$-Bromsubstitutionsproducte schwieriger bilden. Weiter ist aber beobachtet, worden, dass vielfach leichte Bildung gewisser Substanzen mit leichter Rückbildung und entsprechend schwierige Bildung mit schwieriger Zersetzung verbunden sind. Ein besonders eingehend untersuchtes Beispiel hierfür bietet, wie bekannt, die Bildang und Verseifung ron Säureestern.

Es war demnach nach Analogien zu erwarten, dass im vorliegenden Fall das leicht eingetretene, orthoständige Bromatom auch leichter entfernt worden sei, dass also die erhaltenen Reductionsproducte Meta-Bromderivate seien.

War dies richtig, so mussten durch vorsichtige Bromirung der bromfreien Stammsubstanzen isomere Orthoderivate entstehen. Die Darstellung einer bromfreien Verbindung gelang durch andauernde Bebandlung des Dibrom-m-oxypseadocumylalkohols mit Natriumamatgam in alkalischer Lösung. Liess man auf diesen Phenolalkohol die ãquimolekulare Menge Brom in essigsaurer Lösung einwirken, so entstand ein Dibromderivat nach der Gleichung

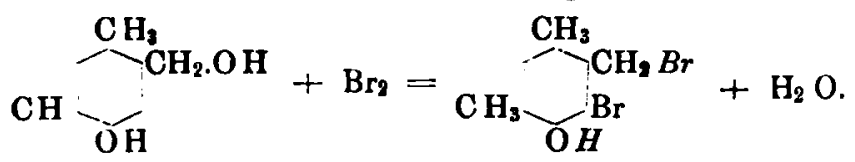

Dieses o-Brom-m-oxypseudocumylbromid verbielt sich in jeder Beziehung analog dem neuen Pseudocumenoltribromid und konnte durch Einwirkung von Natriumacetat und weiter von Essigsäureanhydrid in ein Mono- und ein Diacetat übergefübrt werden.

Die so erhaltenen Verbindungen waren, wie erwartet, nicht identiscb, sondern isomer mit den oben besprochenen Substanzen, und zwar im Sinne folgender Formeln:

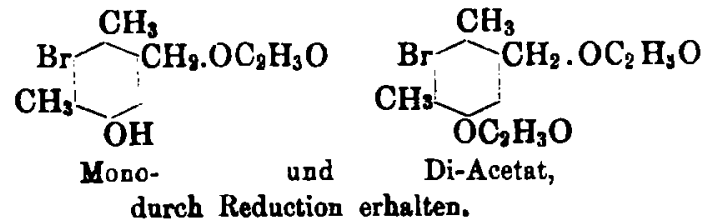




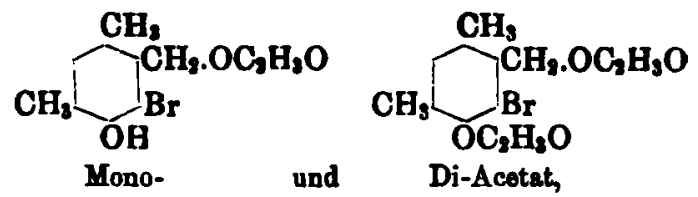

durch Bromirang a. s. w. orhalten.

Durch diese Versuche ist bewiesen, dass in diesem besonderen Fall das zain Hydroxyl metastandige Bromatom fester haftet, als das orthostandige. $O b$ dies allgemein zutriff, müssen weitere Versuche lehren. Sollte dies der Fall sein, so würde diese Erscheinang nicht nur von theoretischem Interesse sein, sondern auch praktisch für die Darstellong mancher, sonst unzuganglicher Substanzen verwerthet werden können.

Das Ausgangematerial für die Darstellung des neuen Pseudocumenoltribromids ist die

Diacetylverbindang des Dibrom-m-oxypseudocumyl-

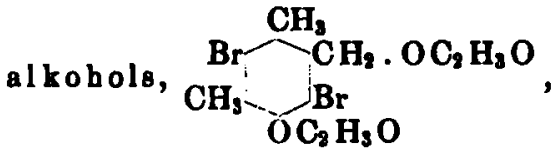

welche entsteht, wenn man das Oxydationsproduct des Dibrompseudocamenols,

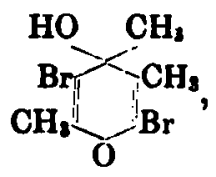

mit dem doppelten Gewicht Essigsalureanhydrids und dem halben Gowicht frisch geschmolzenen Natrinmacetats 7-8 Stunden zum Sieden erhitzt. Man giesst die Reactionstüssigkeit in Wasser, worin sie über Nacht za einer braunen, etwas harzigen Masse erstarrt. Dieses Product wird 2undichst mit kleinen Mengen von Alkohol so lange verrieben, bis es ein hartes Pulver geworden ist, das man anf Thon gat trocknet. Zum Schluss nimmt man den Körper in siedendem Ligroïn auf, kocht eine Stunde mit Thierkohle und lăsst dann krystallisiren. Man erhält durchschnittlich aus 1 Theil Oxykörper $1 / 2$ Theil reines Diacetnt.

Eigenschaften und Anulysen des Körpers sind, wie bei verschiedenen der folgenden Substanzen, schon früher mitgetheilt worden. Sein Schmelzpunkt liegt bei $103-104^{\circ}$. 
Dibrom-m-oxy pseudocumylbromid,

Das eben erwähnte Diacetat löst man in der vierfachen Menge heissen Eisessigs und leitet bei Wasserbadtemperatar lăngere Zeit Bromwasserstoff ein, bei Verarbeitung ron $10 \mathrm{~g}$ Diacetat etwa 4 Stunden. Schon in der Warme beginnt die Abacheidung feiner Nädelchen, and beim Erkalten erstarrt das Ganze zu einem dicken Krystallbrei. Die Verbindung wird abgesangt, mit Eisessig gewascben und aus Ligroïn umkrystallisirt. Die Ausbeute beträgt $8-8.5 \mathrm{~g}$ aus $10 \mathrm{~g}$ Diacetat.

Der Körper bildet lange, seidenglänzende Nadeln vom Schmp. 1280. In Eisessig, Ligroün und Petrolätber ist er in der Kălte schwer löslich, mäseig in kaltem Alkohol; in der Hitze wird er von allen gebriuchlichen organischen Mitteln leicht aufgenommen, von Benzol, Chloroform, Essigester und Aceton schon in der Kälte.

Wie bereits erwähnt, kunn die Substanz im Gegensatz zu dem isomeren Tribromid aus siedendem Alkobol umkrystallisirt werden. Kleine Mengen lassen sich mit sehr verdünntem wässrig-alkobolischem Alkali in Lösung bringen, doch nur für einen Augenblick; dano scheidet sich ein amorphes Zersetzungsproduct aus. Gewöbnliche verdünnte Laugen nebmen das Bromid nicht anf, sondern zersetzen es unter sofort eintretender Braunfärbung.

Wie das bekannte Tribromid vom Schmp. $126^{\circ}$ lässt sich auch das neue Isomere durch Zink und Salzsäure in ätherischer Lősung zu Dibrompseudocumenol reduciren. Man löst das Bromid in Aether, fügt etwas starke Salzsăure und ein paar Stūckchen Zink hinzu und lässt das Gemisch über Nacht stehen. Beim Verdunsten des Aethers hinterbleiben weisse Nadeln, die sich ohne Fãrbung klar in Alkali lösen und nach dem Umkrystallisiren aus Essigsăure den Schmelzpnnkt und die sonstigen Eigenschaften des Dibrompsendocumenols zeigen.

Auch durch kurze Einwirkung ron Zinkstaub auf eine eseigsaure Lōsung des Tribromids wird dieses in gleicher Weise reducirt, wăhrend das alte Isomere unter den gleichen Bedingungen nicht reducirt, sondern in das entsprechende alkaliunlösliche Acetat,<smiles>CCOCC1(Br)C[Bi]C(C)(Br)[Hg]1</smiles>

rom Schmp. $114^{\circ}$ umgewandelt wird.

Kocht man das Tribromid etwa eine Stunde mit dem doppelten Gewicht Easigsanureanhydrid, so wird es in seine Acetylverbindung verwandelt. Vortheilhafter stellt man diese Substanz aus dem 
Diacetat (Schmp. 103-104 ${ }^{\circ}$ ) dar, indem man in eine concentrirte, kalte, essigsaure Lösung dieses Körpers Bromwasserstoffgas bis zur Săttigung einleitet. Es scheidet sich ein dichter Brei von Krystallen ans, die aus Eisesig umkrystallisirt werden.

Dieses Acetat bildet glänzende Nadeln vom Schmp. 105-106". Es ist leicht löslich in Alkohol, Aether, Essigester und Benzol, mässig in Eisessig, Bchwer in Ligroïn und Petroläther.

Behandeit man die Verbindung in heisser essigsaurer Lösung mit Bromwasserstoff, so wird sie natürlich iı das Tribromid (Schmp. 128 verwandelt.

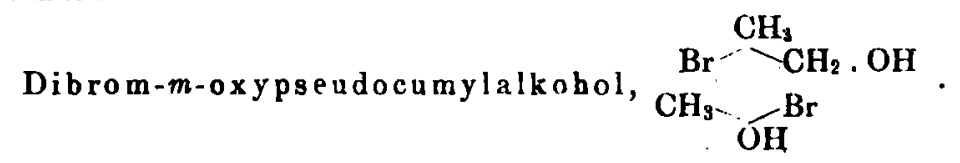

Diese Verbindung kann, wie bereits früher erwübnt, entweder durch Kochen des Tribromids mit wässrigen Aceton, oder dureh Verseifung des Diacetats vom Schmp. $103-104^{\circ}$ erhalten werden. Die erste Methode liefert schlechte, die zweite, bei rorsichtigem Arbeiten, befriedigende Ausbeuten.

Man löst das Diacetat in heissem Alkohol und fügt tropfenweise verdūnnte Natronlauge hinzu, bis eine Probe der Flüssigkeit beim Verdünnen mit Wasser klar bleibt. Hat man ganz reines Diacetat angewendet, so ist die Flüssigkeit nach beendeter Verseifung nur hellorange gefürbt; verwendet man aber ein unreines Präparat oder setzt zu viel Alkali hinzu, so tritt eine tiefdunkelrothe Färbung ein, und man kann nur mit grossen Verlusten das reine Verseifungsproduct gewinnen. Die schwach alkalische Flüssigkeit wird vorsichtig mit verdünnter Essigsăure angesäuert, der Niederschlag abfiltrirt, gewaschen, getrocknet und aus Benzol oder Chloroform; wenn nöthig unter Zusatz von Thierkohle, mehrfach umkrystallisirt.

In völlig reinem Zustand schmilat der Phenolalkohol scharf bei $154^{\circ}$ and stellt glänzende, weisse Nadeln dar. In Aether, Alkohol, Aceton and Essigester ist er leicht löslich; in Eisessig mässig, in Benzol, Chloroform und Ligroïu schwer. In concentrirter Schwefelsäure lōst er sich farblos auf.

$$
\begin{aligned}
& 0.1017 \mathrm{~g} \text { Sbst.: } 0.2139 \mathrm{~g} \mathrm{CO}_{2}, 0.0514 \mathrm{~g} \mathrm{H}_{2} \mathrm{O} \text {. } \\
& \mathrm{C}_{9} \mathrm{H}_{40} \mathrm{O}_{2} \mathrm{Br} . \quad \text { Ber. C 34.84, } \mathrm{H} \mathrm{3.23,} \mathrm{Br} 51.6 \mathrm{I} \text {. } \\
& \text { Gef. 》35.13, *3.44, » 51.14. }
\end{aligned}
$$

Kochendes Essigsăureanhydrid verwandelt den Alkohol in dir Diacetylverbindung rom Schmp. 103-104 zurück. Bromwasserstoff, in seine warme essigsaure Lösung eingeleitet, regenerirt das Tribromid (Schmp. 1280).

Die Aether dieses Alkohols entstehen durch Umsetzung des Tribromids mit den betreffenden Alkoholen. Man kocht zu diesem 
Zweck das Bromid mit den Alkoholes, bis eine ausgefillte Probe des Reactionsproductes sich ohne Brounung in verdünntem Alkali 18at, was bei Verarbeitung von $5 \mathrm{~g}$ Bromid nach etwa 2 Stonden der Fall za sein plegt. Man fällt dann die Lossang mit Waoser und krystallisirt den Niederschlag um.

Der Dibrom-m-oxy pseudocumylmethylather, $\mathrm{C}_{6}\left(\mathrm{CH}_{2}\right)_{2} \mathrm{Br}_{2}$. (OH). $\mathrm{CH}_{2} . \mathrm{OCH}_{3}$, vom Schmp. $106^{\circ}$ ist schon beschrieben worden. Seine Acetylverbindung, die durch Kochen mit Eseigowareanhydrid leicht erhalten wird, krystallisirt ans Methylalkohol in feinen, weissen Nádelchen, die bei $80-81^{\circ}$ schmelzen und in ullen gebriachlicben organischen Mitteln leicht löslich sind.

$0.1232 \mathrm{~g}$ Sbst: $0.1270 \mathrm{~g} . \mathrm{lgBr}$.

$\mathrm{C}_{19} \mathrm{H}_{14} \mathrm{O}_{3} \mathrm{Br}$. Ber. $\mathrm{Br}$ 43.71. Gef. $\mathrm{Br} 43.86$.

Der entsprechende $\mathrm{A}$ e th yla ther, $\mathrm{C}_{6}\left(\mathrm{CH}_{3}\right)_{2} \mathrm{Br}(\mathrm{OH}) \cdot \mathrm{CH}_{2} . \mathrm{OC}_{2} \mathrm{H}_{3}$, schmilzt bei 63-640. Glagglinzende Priomen ans Petrolkther. Leicht löslich.

0.1607 g Sbst.: $0.2298 \mathrm{~g} \mathrm{CO}, 0.0639 \mathrm{~g} \mathrm{H}_{3} \mathrm{O}$.

$0.1665 \mathrm{~g}$ Sbst.: $0.1851 \mathrm{~g} \mathrm{AgBr}$.

$\mathrm{C}_{11} \mathrm{H}_{14} \mathrm{O}_{2} \mathrm{Br}$. Ber. C 39.05, H 4.14, Br 47.33.

Gef. 38.95 , 4.42, 47.31 .

Ueber die Darotellung, Eigenschaften und Umwandlungen der bei $10 t^{0}$ schmelzenden Monoacetylverbindung des Dibrom-m-oxypseudocumplalkohols,<smiles>CCO[C@H](Br)[C@@H](Br)CBr</smiles>

ist bereits kurz berichtet worden. Nachzatragen ist, dass die Ueberführung des Tribromids in diese Verbindang durch Kochen mit Natriumacetat in Eisessig bei Verarbeitung von $5 \mathrm{~g} 2-3$ Stunden in Anspruch nimmt, und duss das Acetat in organischen Mitteln, mit Ausnabme von Ligroin und Petrolather, leicht löslich ist.

\section{Reductions versuche.}

Ùm zu der bromfreien Stammsabstanz der beachriebenen Verbindnngen zl gelangen, wurde in erater Linie die am leichtesten zagingliche Diacetylverbindang rom Schmp. 103-1040 der Reduction nnterworfen.

a) Reduction mit Natrinmamalgam in essigsaurer Lösung. $5 \mathrm{~g}$ reines Diacetat wurden in kalter 75-procentiger Essigelure gelöst und im Laufe ron 2 Tagen mit etwa $500 \mathrm{~g}$ 2-procentigem Natriumamalgam versetzt. Auf Zusatz ron Wasser schied sich ein Unl aus, das rasch erstarrte. Nach dem Trocknen auf Thon wurde die Substans mehrfach an Petrolather umkrystallisirt, bis der Schmelzpunkt bei $65^{\circ}$ constant geworden war. 
Die Substanx bildet schone, farblose Prismen und int im Allgemeinen sebr leicht 16slich. Eine Brombestimmung ergab, daes der Korper noch ein Bromatom entbalt; er ist daher, wie in der Einleitung anogefubrt worden iat, als Diacetylverbindung des $m$-Bromm-oxypsendocumylalkohols,

aufzofussen.

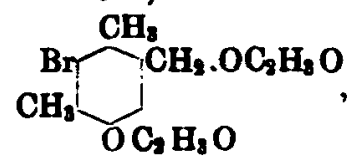

$0.1384 \mathrm{~g}$ Sbst.: $0.0807 \mathrm{~g} \mathrm{AgBr}$.

$\mathrm{C}_{13} \mathrm{H}_{15} \mathrm{O}$, Br. Ber. Br 25.40. Gef. Br 25.74.

b) Reduction mit Zinkstaub in essigsaurer Losung. $5 \mathrm{~g}$ Diacetat warden in 75-procentiger Essigekare gelöst and einen Tag lang mit Zinkstanb gekocht. Aus der vom Zink abgegossenen Flüesigkeit fillte Wasser ein röthliches $\mathrm{Oel}$, das mit Aether anfgenommen warde. Die Losung warde mit Wasser gewaschen, getrocknet, dann der Aether abdestillirt und der Rückstand nochmals 2 Standen mit Essigabare and Zinkstaub erbitzt. Das nunmehr erbaltene Reductionsprodact erstarrte aach gleicher Bebandlung und warde mehrfach aus Ligroin umkrystallisirt.

Diese Monoacetylverbindung des $n$-Brom-m-oxypseudocumylalkobols von der Formel<smiles>CCO[C@H](C)[C@H](C)O</smiles>

Krystalliairt in feineo Nádelchen, schmilzt bei $104^{\circ}$ und ist in den meisten organischen Mitteln leicht löslich; nur von Ligroīn und Petrolather wird sie etwas schwerer aufgenommen. Als Phenol lōst sie sich in whssrigen Altalien.

$0.1838 \mathrm{~g}$ Sbst.: $0.1275 \mathrm{~g} \mathrm{AgBr}$.

$\mathrm{C}_{21} \mathrm{H}_{13} \mathrm{O}_{3}$ Br. Ber. Br 29.30. Gef. Br 29.51 .

Beide Acetylverbindungen wurden durch gelinde Digestion mit alkoholischem Natron zum

m-Brom-m-oxypseudocumylalkohol,<smiles>C[C@H]1C[C@H](O)[C@H](O)C1</smiles>

vereeift.

Dieser Körper, der durch Umkrystallisiren ans Benzol oder verdūnntem Methylalkohol leicht gereinigt werden kann, bildet glänzende, weisse Blattchen vom Schmp. 1640. Br ist leicht 18slich in Alkohol, Aether, Aceton, Eisessig und Essigester; schwer in Benzol, Chloroform, Ligroin und Petroläther. 
$0.1434 \mathrm{~g}$ Sbst.: $0.2456 \mathrm{~g} \mathrm{CO}, 0.0658 \mathrm{~g} \mathrm{H}_{3} \mathrm{O}$.

$0.1681 \mathrm{~g}$ Sbst.: $0.1360 \mathrm{~g} \mathrm{AgBr}$.

$$
\begin{aligned}
& \mathrm{C}_{9} \mathrm{H}_{11} \text { O,Br. Ber. C 46.75, H 4.76, Br 34.63. } \\
& \text { Gef. 46.71, 5.10, } \$ 34.42 \text {. }
\end{aligned}
$$

Der Alkohol kann in die beiden zuror beschriebenen Acetylverbindungen zurückverwandelt werden.

Um das Monoacetat zu erhalten, löst man den Alkohol in wenig Eisessig, leitet kurze Zeit Salzsăuregas ein, concentrirt die Lösung etwas und fallt dann mit Wasser. Der Niederschlag schmilzt nach dem Umkrystallisiren ans Petrolăther constant bei $104^{\circ}$.

Das Diacetat rom Schmp. $65^{\circ}$ wird in der gewöhnlichen Weise durch längeres Kochen des Alkohols mit Essigsäureanhydrid gewonnen.

Die bromfreie Stammsubstanz der eben beschriebenen Verbindungen, der

$$
\text { m-Oxypseudocumylalkohol, }
$$

entsteht, wenn der zweifach gebromte Phenolalkohol (Schmp. 154-1550) längere Zeit in alkalischer Lösung mit Natriumamalgam behandelt wird.

$10 \mathrm{~g}$ Dibromphenolalkohol wurden in verdünnter kalter Natronlauge gelöst und im Lanfe von 10 Tagen allmăhlich mit etwa $5 \mathrm{~kg}$ 2 - procentigem Natriumamalgam versetzt. Die Lōsung wurde daranf mit Essigsäure angesăuert und mehrfach mit Aether ausgeschüttelt. Die getrockneten ätherischen Auszüge hinterliessen beim Verdunsten eine weisse Masse, die aus Benzol oder heissem Wasser umkrystallisirt wurde. Die Ausbeute an reinวr Substanz betrug $4.5 \mathrm{~g}$, d. h. etwa $90 \mathrm{pCt}$. der Theorie.

Der Körper krystallisirt in perlmutterglänzenden Blăttcben, die scharf bei $153^{\circ}$ schmelzen. Von concentrirter Schwefelsăure wird die Substanz, ebenso wie ibr zuvor beschriebenes Monobromderivat, dunkelbrann gefärbt und beim Erwarmen mit gleicher Farbe gelőst. In kaltem Wasser, Benzol, Ligroĩn und Petrolăther ist der Alkohol schwer löslich, leicht oder ziemlich leicht in den übrigen organischen Mitteln.

$0.1466 \mathrm{~g}$ Sbst.: $0.3824 \mathrm{~g} \mathrm{CO}_{2}, 0.1068 \mathrm{~g} \mathrm{H}_{2} \mathrm{O}$.

$\mathrm{C}_{3} \mathrm{H}_{12} \mathrm{O}_{2}$. Ber. C 71.06, H 7.90 .

Gef. $71.12, \quad 8.10$.

Lässt man auf die fein gepulverte Substanz direct Brom tropfen, so tritt eine heftige Reaction ein, deren Product das Pseudocumenoltribromid rom Schmp. $128^{\circ}$ ist. Bebandelt man aber den Alkohol in essigsaurer Lösung vorsichtig mit Brom, so entsteht das um ein Bromatom ärmere 


\section{4}

o-Brom-m-oxypseudocumylbromid,

$5 \mathrm{~g} m$-Oxypseudocumylalkohol werden in kaltem Eisessig gelöst und tropfenweise mit einer Mischung von 1 Vol. Brom und 9 Vol. Eisessig versetzt, bis die Farbe des Broms nicht mehr verschwindet. Man dunstet die Lösung alsdann etwas ein, filtrirt die ausgeschiedenen Krystalle ab und krystallisirt sie aus Ligroïn um.

Die Substanz bildet lange, seidenglänzende Nadeln vom Schmp. $116^{\circ}$, die sich mit Ausnahme von Ligroïn und Petroläther in den gebräuchlichen organischen Mitteln leicht lösen. Wie das Tribromid, wird der Körper von verdünntem Alkali augenblicklich unter Braunfürbung zersetzt. Das Bromatom der Seitenkette wird von Alkoholen, wăssrigem Aceton, Natriumacetat u. s. w. nicht sofort, sondern erst nach längerem Kochen herausgenommen, wobei alkalilösliche Verbindungen entstehen.

0.1385 g Sbst.: $0.1834 \mathrm{~g} \mathrm{CO}_{8}, 0.0444 \mathrm{~g} \mathrm{H}_{2} \mathrm{O}$.

$0.1924 \mathrm{~g}$ Sbst.: $0.2472 \mathrm{~g} \mathrm{AgBr}$.

$$
\mathrm{C}_{2} \mathrm{H}_{10} \mathrm{OBr} \text {. } \begin{aligned}
& \text { Ber. C } 36.74 \text {, H } 3.40, \text { Br } 54.42 . \\
& \text { Gef. \$ 36.12, 》 } 3.56 \text {, } 54.68 .
\end{aligned}
$$

Aus $10 \mathrm{~g}$ Phenolalkohol warden durchschnittlich nur $8 \mathrm{~g}$ reines Dibromid oder etwa $42 \mathrm{pCt}$. der theoretischen Ausbente erhalten.

Zar Umwandlung in die Monoacetylverbindung des o-Bromm-oxypseudocumylalkohols,<smiles>CCO[C@@H](C)[BH-]1CCC(C)O1</smiles>

kocht man äquimolekulare Mengen des Bromids und Natriumacetats etwa 2 Stunden in essigsaurer Lösung, bis eine Probe des Reactionsproductes von Alkalien nicht mehr braun gefärbt wird. Man fállt dann die Flüssigkeit mit Wasser, filtrirt den Niederschlag ab und krystallisirt ibn nach dem Trocknen aus Ligroïn um.

Weisse Prismeu vom Schmp. $103^{\circ}$. Nur in Ligroïn und Petrolather schwer, sonst leicht löslich.

$0.1792 \mathrm{~g}$ Sbst.: $0.1232 \mathrm{~g} \mathrm{AgBr}$.

$\mathrm{C}_{11} \mathrm{H}_{13} \mathrm{O}_{3} \mathrm{Br}$. Ber. Br 29.30. Gef. Br 29.25 .

Der Körper hat fast den gleichen Schmelzpunkt wie die isomere Verbindung mit metaständigem Bromatom; das Gemisch beider schmilzt jedoch schon bei $75^{\circ}$, woraus die Verschiedenheit der Substanzen folgt.

Darch Kochen mit Essigsäureanibydrid wird der Körper in die entsprechende Diacetylverbindung verwandelt, die man gleichfalls 
durch Umkrystallieiren aus Ligroïn reinigt. Sie schmilzt bei $57^{\circ}$; das Gemisch mit dem isomeren Diacetat rom Schmp. $65^{\circ}$ bei $48^{\circ}$. Ihre Lößlichkeitoverbältnisse gleichen denen der Monoacetylverbindung. $0.1027 \mathrm{~g}$ Sbst. : $0.0624 \mathrm{~g} \mathrm{AgBr}$.

$\mathrm{C}_{13} \mathrm{H}_{15} \mathrm{O}_{4}$ Br. Ber. Br 25.40. Gef. Br 25.86.

Durch Verseifung wird die Verbindung in den zugehörigen 0-Brom. m-oxypseudocumylalkohol übergeführt, doch konnte die Substanz wegen Mangels an Material nicht vollkommen rein erhalten werden.

Heidelberg, Universitätslaboratoriam.

510. K. Auwers und J. Broioher: Zur Kenntnies der Oxydationsproducte von Phenolen und Phenolbromiden.

(Eingegangen am 28. November.)

Die im Folgenden beschriebenen Versuche wurden angestellt, um die in den vorstehenden Arbeiten mitgetheilten Beobachtungen über das Verhalten der Oxykōrper aus dem Dibrom- und TribromDerivat des Pseudocumenols auf ihre Allgemeinheit zu prüfen.

I.

Es ist berichtet worden, dass das Oxydationsproduct des Dibrompseudocumenols (I) bein Kochen mit Essigsäureanbydrid allein eine Monoacetylverbindung (II) liefert, wâhrend bei Zusatz von entwässertem Natriumacetat die Diacetylverbindung eines Meta-Phenolalkobols (III) entsteht:

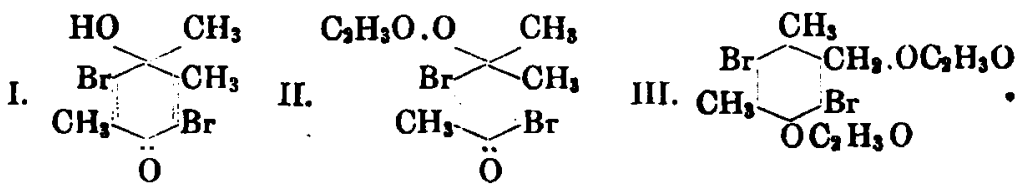

Vergeblich haben wir uns bemüht, den Oxykörper des analogen Dibrommesitols') (IV) in gleicher Weise in ein Diacetat umzuwandeln, denn auch wenn der Oxykörper mit Essigsăureanhydrid und Natriumacetat in Rohr auf böhere Temperatur erbitst warde, bildete sich doch nur die Monoacetylverbindung (V), die schon beim blossen Kochen mit Essigsăureanbydrid gewonnen wird.<smiles>CC(=O)C(C)(Br)C(C)(Br)C(C)(O)C(Br)(Br)C(C)=O</smiles>

b) Vergl. Auwers.und Rapp, Ann. d. Chem. 302, 167. 